\title{
NEW STRESS INTENSITY FACTOR SOLUTIONS FOR AN ELLIPTICAL CRACK IN A PLATE
}

\author{
Patrick Le Delliou \\ Electricité de France R\&D \\ Les Renardières \\ 77818 Moret-sur-Loing Cedex \\ France \\ Phone: 33160736403 \\ Fax: 33160736559 \\ E-mail: Patrick.le-delliou@edf.fr
}

\author{
Bruno Barthelet \\ Electricité de France \\ Nuclear Power Operations \\ Site Cap Ampère \\ 93282 Saint Denis Cedex \\ France
}

\begin{abstract}
Crack assessment in engineering structures relies first on accurate evaluation of the stress intensity factors. In recent years, a large work has been conducted in France by the Atomic Energy Commission to develop influence coefficients for surface cracks in pipes. However, the problem of embedded cracks in plates (and pipes) which is also of practical importance has not received so much attention. Presently, solutions for elliptical cracks are available either in infinite solid with a polynomial distribution of normal loading or in plate, but restricted to constant or linearly varying tension.
\end{abstract}

This paper presents the work conducted at EDF R\&D to obtain influence coefficients for plates containing an elliptical crack with a wide range of the parameters : relative size $(2 \mathrm{a} / \mathrm{t}$ ratio), shape $(\mathrm{a} / \mathrm{c}$ ratio) and free surface proximity ( $a / d$ ratio where $d$ is the distance from the center of the ellipse to the closest free surface). These coefficients were developed through extensive 3D finite element calculations : 200 geometrical configurations were modeled, each containing from 18000 to 26000 nodes. The limiting case of the tunnel crack $(\mathrm{a} / \mathrm{c}=0)$ was also analyzed with 2D finite element calculation (50 geometrical configurations). The accuracy of the results was checked by comparison with analytical solutions for infinite solids and, when possible, with solutions for finite-thickness plates (generally loaded in constant tension).

These solutions will be introduced in the RSE-M Code that provides rules and requirements for in-service inspection of French PWR components.

Keywords: Stress intensity factor (SIF), influence coefficient, plate, elliptical crack

\section{NOMENCLATURE (SEE FIGURE 1)}

a

c

d

e

$\mathrm{E}$

$\mathrm{E}(\mathrm{k})$

$\mathrm{i}_{\mathrm{j}}$

$\mathrm{k}$

Semi-minor axis of ellipse

Semi-major axis of ellipse

Distance from the closest free surface to the center of the ellipse

Distance from the plate mid plane to the center of the ellipse

Young's modulus

Complete elliptic integral of the second kind

Influence coefficient for the $j$ th degree $\quad(0 \leq \mathrm{j} \leq 3)$

Modulus of Jacobian elliptic functions, with $\mathrm{k}^{2}=1-(\mathrm{a} / \mathrm{c})^{2}$ 


$\begin{array}{ll}\mathrm{K}_{\mathrm{I}} & \text { Mode I stress intensity factor } \\ \mathrm{t} & \text { Plate thickness } \\ \phi & \text { Parametric angle defining a location on the crack front } \\ v & \text { Poisson's ratio } \\ \sigma_{\mathrm{j}} & \text { coefficient for the } j \text { th degree of the polynomial stress distribution }\end{array}$
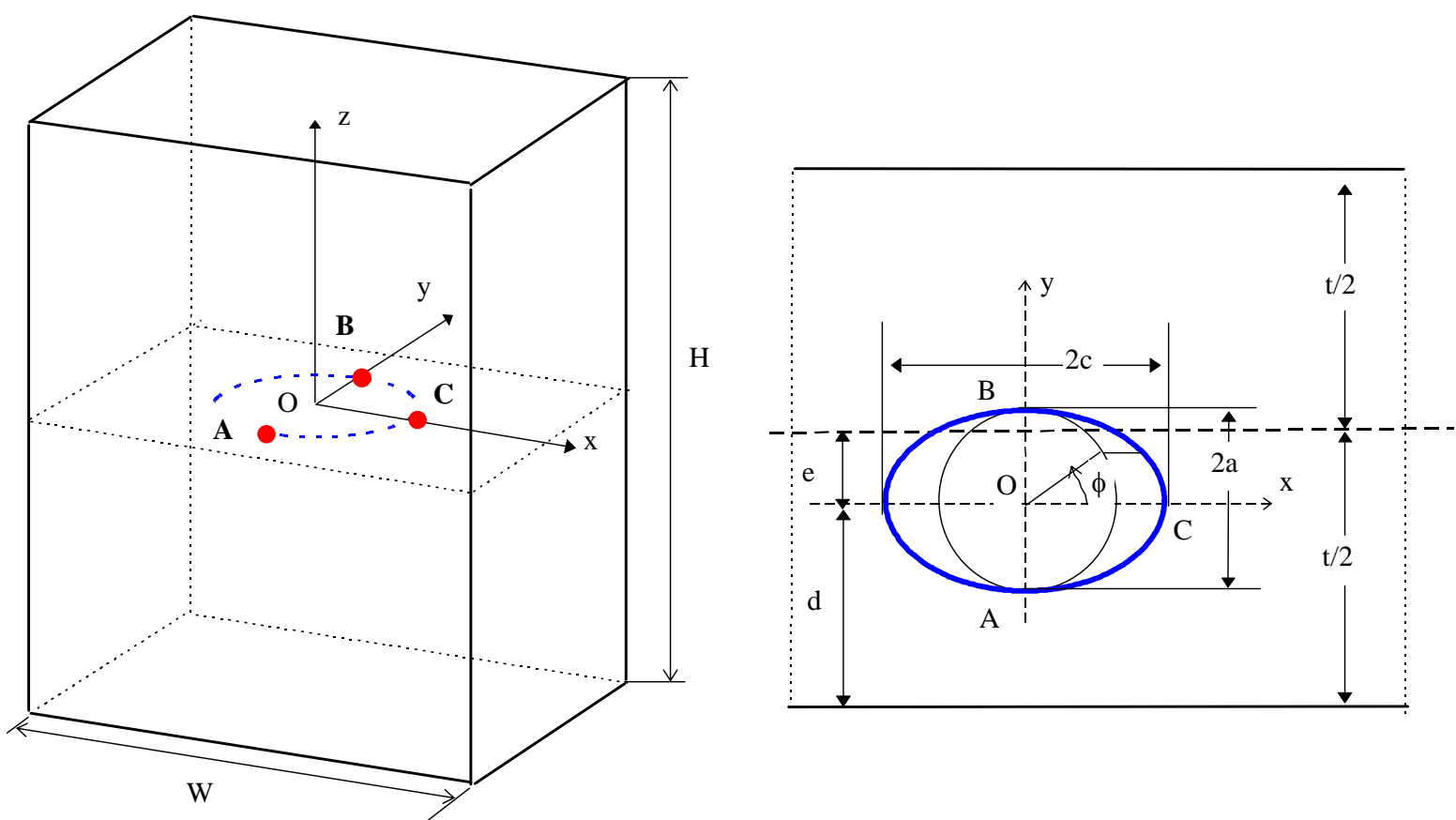

Figure 1: An elliptical crack in a plate: definition of the geometrical parameters.

\section{INTRODUCTION}

Crack assessment in engineering structures relies first on accurate evaluation of the stress intensity factors. In recent years, a large work has been conducted in France by the Atomic Energy Commission to develop influence coefficients for surface cracks in pipes (Chapuliot, 1998, 1999). These results have been included in the RSE-M Code (RSE-M, 2000), that provides rules and requirements for in-service inspection of French PWR components. However, the problem of embedded cracks in plates (and pipes) which is also of practical importance has not received so much attention. Presently, solutions for elliptical cracks are available either in infinite solid with a polynomial distribution of normal loading (Irwin, 1962, Shah, 1971, Isida, 1976) or in plate (Isida, 1956, 1966, 1984, Shah, 1971, 1973, 1974, Nisitani, 1974, Shiratori, 1992, ASME, 1998), but restricted to constant or linearly varying tension. Most of these solutions can be found in the compilation edited by Murakami (1992).

The objective of this study was to calculate accurate stress intensity factors for embedded elliptical cracks in plates for a wide range of the geometrical parameters defined hereunder :

o the relative crack size ( $2 \mathrm{a} / \mathrm{t}$ ratio), ranging from 0.05 to 0.5 ,

o the shape of the ellipse (a/c ratio), ranging from 1 (penny-shaped crack) to 0 (tunnel crack),

$o$ the free surface proximity $(\mathrm{a} / \mathrm{d}$ ratio) where $\mathrm{d}$ is the distance from the center of the ellipse to the closest free surface, ranging from $2 \mathrm{a} / \mathrm{t}$ (centered crack) to 0.95 .

The influence coefficients $\left(i_{0}\right.$ to $i_{3}$ ) were developed for a third-order polynomial stress distribution in the thickness direction expressed in the local coordinate system Oxyz (figure 1) as follows :

$$
\sigma_{\mathrm{zz}}\left(\frac{\mathrm{y}}{\mathrm{a}}\right)=\sum_{\mathrm{j}=0}^{3} \sigma_{\mathrm{j}}\left(\frac{\mathrm{y}}{\mathrm{a}}\right)^{\mathrm{j}}
$$

Then, the stress intensity factor at the point of elliptic angle $\phi$ is expressed with the coefficients $\sigma_{j}$ and the influence coefficients $i_{j}$ by the relationship : 


$$
\mathrm{K}_{\mathrm{I}}(\phi)=\sqrt{\pi \mathrm{a}} \sum_{\mathrm{j}=0}^{3} \sigma_{\mathrm{j}} \mathrm{i}_{\mathrm{j}}(\phi)
$$

255 finite element calculations were performed to achieve this goal :

o 204 three-dimensional $\mathrm{FE}$ calculations for $\mathrm{a} / \mathrm{c}=1,0.5,0.25$ and 0.125 ,

o 51 two-dimensional FE calculations for $\mathrm{a} / \mathrm{c}=0$ (tunnel cracks).

\section{FINITE ELEMENT ANALYSIS}

\subsection{Mesh generation}

The meshes were made with a parametric procedure using Gibi, a powerful meshing software developed by CEA (French Atomic Energy Commission). With this procedure, the generation of a new mesh takes only a few minutes, as the work is limited to the introduction of the geometrical parameters. Isoparametric quadratic elements are used (either 20 node solid elements or 8 node elements depending on the FE model).

The mesh of a plate containing an elliptical crack was derived from a procedure aiming to model a semi-elliptical surface crack in a plate. The mesh of a plate (thickness : $t / 2$ - e) containing a semi-elliptical crack (depth : a and length : $2 \mathrm{c}$ ) is created. This mesh is duplicated by a symmetry with respect to the plane $y=0$. This copy is added to the original mesh and a volume whose thickness is $2 \mathrm{e}$ is finally added to complete the mesh. The symmetries are taken into account, so only a quarter of the plate is modeled. The width $\mathrm{W}$ and the height $\mathrm{H}$ of the plate are chosen large enough to assume that the plate is of infinite size. They are 65 nodes along the crack front. Moreover, these nodes are equally spaced with regard to the parametric angle $\phi$, due to the elliptical transformation used to create the crack tip mesh. A typical mesh is shown in figure 2 . The meshes contain between 20,000 and 30,000 nodes, depending on the geometrical parameters.

\subsection{Description of the calculations}

The calculations were made with the finite element program Code_Aster, developed by EDF. A linear elastic material with a Young's modulus $\mathrm{E}=200 \mathrm{GPa}$ and a Poisson's ratio $v=0.3$ was considered. The fixed boundary conditions were applied to the planes $\mathrm{x}=0$ and $\mathrm{z}=0$ according to the symmetries involved in the geometry. For each crack geometry, four types of loading were applied directly on the crack surface, with the following pressure distributions :

$$
\sigma_{\mathrm{zz}}\left(\frac{\mathrm{y}}{\mathrm{a}}\right)=\sigma_{0}\left(\frac{\mathrm{y}}{\mathrm{a}}\right)^{\mathrm{j}} \quad \text { with } 0 \leq \mathrm{j} \leq 3
$$

The energy release rate $\mathrm{G}$ was calculated at each node of the crack front by the G-Theta method, based on a domain integral technique (Wadier, 1989). $\mathrm{K}_{\mathrm{I}}$ was calculated from $\mathrm{G}$ assuming plane strain conditions and equation (2) was used to derive the influence coefficient from $\mathrm{K}_{\mathrm{I}}$, so the influence coefficient is given by :

$$
\mathrm{i}_{\mathrm{j}}(\phi)=\frac{1}{\sigma_{0}} \sqrt{\frac{\mathrm{G}_{\mathrm{j}}(\phi) \mathrm{E}}{\pi \mathrm{a}\left(1-v^{2}\right)}}
$$




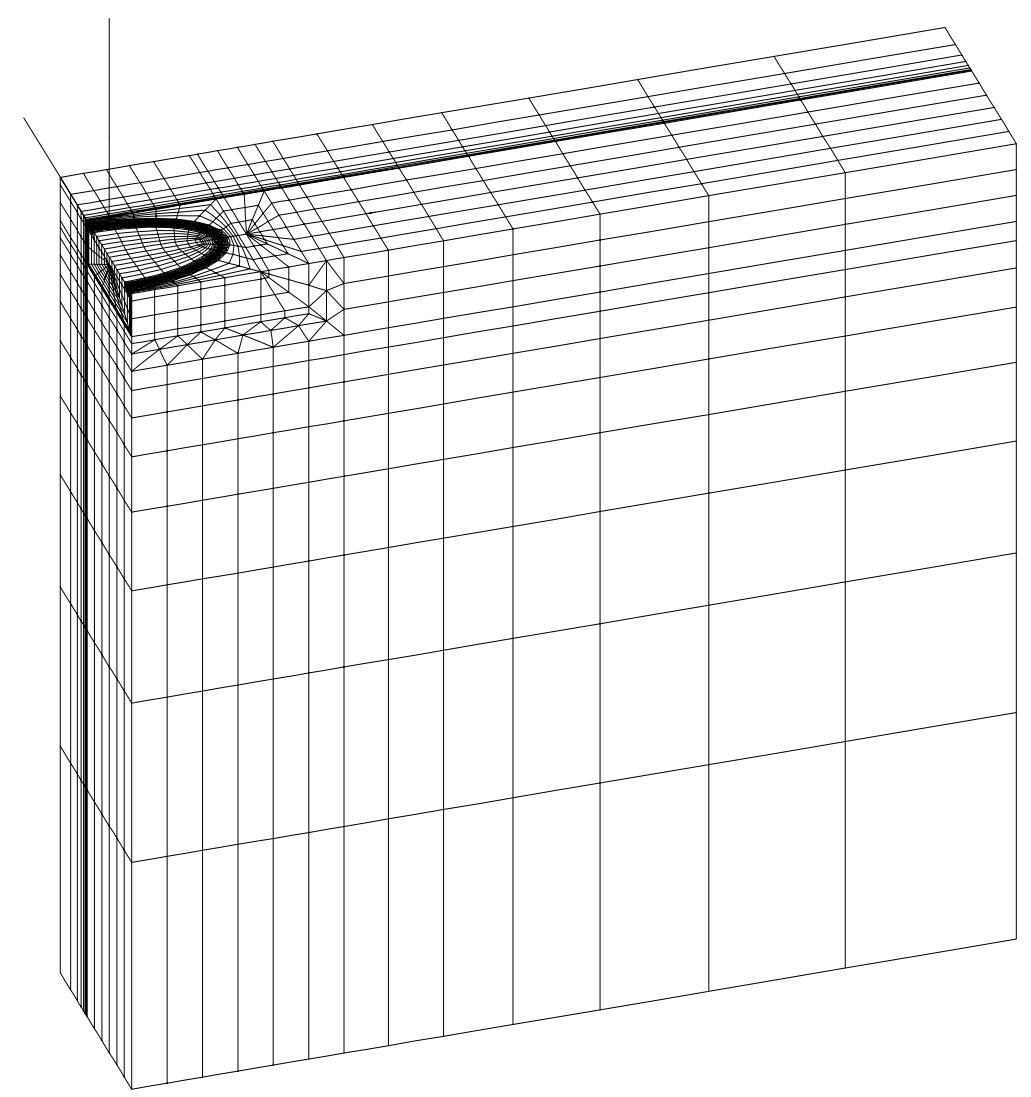

Figure 2: Typical finite element mesh $2 a / t=0.5, a / c=0.5, a / d=0.375$ (23,173 nodes)

\section{RESULTS}

\subsection{Tables of influence coefficients}

Influence coefficients have been gathered in twelve tables, i.e. at 3 points of the crack front (A, B and C) and for 4 loading degrees. These tables are given in this paper (Tables 1 to 12).

\subsection{Validation}

The comparison between the present results and those found in the literature is made by calculating the relative difference by the relation :

$$
\operatorname{Diff}(\%)=100\left(\frac{\mathrm{i}_{\text {Present }}}{\mathrm{i}_{\text {Lit }}}-1\right)
$$

For tunnel cracks $(\mathrm{a} / \mathrm{c}=0)$ ), the exact solution (Isida, 1976) for a crack in an infinite solid $(2 \mathrm{a} / \mathrm{t}=0)$ was used to assess the results for $2 \mathrm{a} / \mathrm{t}=0.05$. The differences range from $-0.1 \%$ for $\mathrm{i}_{0}$ to $-0.5 \%$ for $\mathrm{i}_{3}$. For a crack in a finite-thickness plate, approximate solutions are only available for the constant loading (Isida, 1966) and for a centered crack submitted to a linear loading (Isida, 1956, Benthem, 1972). For the centered crack, a specific study was conducted for crack sizes up to $2 \mathrm{a} / \mathrm{t}=0.8$. For the constant loading, the maximum difference with (Isida, 1966) was $-0.10 \%$. For the linear loading, the maximum difference with (Isida, 1956) was $-1.2 \%$ (for $2 \mathrm{a} / \mathrm{t}=0.7$ ). The solution (Benthem, 1972) seems to give too high values when $2 \mathrm{a} / \mathrm{t}$ is larger than 0.5 . 
For elliptical cracks (a/c > 0), the exact solutions (Green, 1950, Irwin, 1962, Shah, 1971) for a crack in an infinite solid $(2 \mathrm{a} / \mathrm{t}=0)$ were used to assess the results for $2 \mathrm{a} / \mathrm{t}=0.05$. For the constant loading, the influence coefficient $\mathrm{i}_{0}$ is given by :

$$
\mathrm{i}_{0}(\phi)=\frac{1}{\mathrm{E}(\mathrm{k})}\left[\sin ^{2} \phi+\left(\frac{\mathrm{a}}{\mathrm{c}}\right)^{2} \cos ^{2} \phi\right]^{\frac{1}{4}}
$$

For the linear loading, the influence coefficient $i_{1}$ is given by the expression (Shah, 1971) :

$$
\mathrm{i}_{1}(\phi)=\frac{1}{3 \mathrm{E}_{2}(\mathrm{k})} \sin \phi\left[\sin ^{2} \phi+\left(\frac{\mathrm{a}}{\mathrm{c}}\right)^{2} \cos ^{2} \phi\right]^{\frac{1}{4}}
$$

where $\mathrm{E}_{2}(\mathrm{k})$ is an elliptic integral defined by :

$$
\mathrm{E}_{2}(\mathrm{k})=\frac{1}{3 \mathrm{k}^{2}}\left[\left(1+\mathrm{k}^{2}\right) \mathrm{E}(\mathrm{k})-\left(1-\mathrm{k}^{2}\right) \mathrm{K}(\mathrm{k})\right]
$$

In these expressions, $\mathrm{K}(\mathrm{k})$ and $\mathrm{E}(\mathrm{k})$ are respectively the complete elliptic integrals of the first kind and of the second kind.

For the quadratic loading, the influence coefficient $i_{2}$ is given by the expression (Wu, 2000) (the Kassir and Sih solution's is identical, expect that the constant term was omitted) :

$$
\mathrm{i}_{2}(\phi)=\mathrm{i}_{0}(\phi)\left[0,2-\frac{2 \mathrm{~J}_{22} \mathrm{~J}_{00}}{15 \Delta} \cos ^{2} \phi+\frac{2\left(\mathrm{~J}_{20}-\mathbf{J}_{22}\right) \mathrm{J}_{00}}{15 \Delta} \sin ^{2} \phi\right]
$$

where :

$$
\begin{aligned}
& \mathrm{i}_{0} \text { is the influence coefficient for the constant loading (Eq. 6), } \\
& \mathbf{J}_{00}, \mathbf{J}_{20} \text { et } \mathbf{J}_{22} \text { are elliptical integrals, } \\
& \Delta=\mathbf{J}_{20} \mathbf{J}_{02}-\mathbf{J}_{00} \mathbf{J}_{22}
\end{aligned}
$$

The elliptical integrals are defined hereafter :

$$
\begin{aligned}
& \mathrm{J}_{00}=\mathrm{E}(\mathrm{k}) \quad \mathrm{J}_{02}=\mathrm{E}_{2}(\mathrm{k}) \quad \mathrm{J}_{20}=\mathrm{J}_{00}-\mathrm{J}_{02} \\
& \mathrm{~J}_{22}(\mathrm{k})=\frac{1}{15 \mathrm{k}^{4}}\left[2\left(\mathrm{k}^{4}-\mathrm{k}^{2}+1\right) \mathrm{E}(\mathrm{k})-\left(\mathrm{k}^{4}-3 \mathrm{k}^{2}+2\right) \mathrm{K}(\mathrm{k})\right]
\end{aligned}
$$

At point $\mathrm{A}$, the differences were comprised between $-0.2 \%$ (constant loading) and $-0.4 \%$ (quadratic loading). At point $\mathrm{C}$, the differences were comprised between $0.2 \%$ (constant loading) and $-4 \%$ (quadratic loading). At this point, the difference mainly depends on the a/c ratio, as it corresponds to the sharpest curvature of the ellipse.

For a crack in a finite-thickness plate, most of the solutions are relative to the constant loading (Shah, 1973, Nisitani, 1974, Isida, 1984, Guozhong, 1996) and to the linear loading (Shah, 1971, 1974). Influence coefficients up to the third-degree are given in (Shiratori, 1992) for a crack with $2 \mathrm{a} / \mathrm{t}=0.2$. The accuracy of all these approximate solutions is difficult to assess. On the overall, the accuracy of the present results is estimated better than $0.5 \%$ at points $\mathrm{A}$ and $\mathrm{B}$, and ranging between $0.5 \%$ and $5 \%$ at point $\mathrm{C}$, depending on the loading degree and the $\mathrm{a} / \mathrm{c}$ ratio.

\section{CONCLUSIONS}

Two and three-dimensional finite element analyses have been conducted to calculate influence coefficients up to the third order for elliptical cracks embedded in plates, for a wide range of the geometrical parameters defining the crack : size, shape and relative distance to the closest free surface. The accuracy of these coefficients has been checked by comparison with exact or approximate solutions available in the literature. 


\section{REFERENCES}

ASME Boiler and Pressure Vessel Code (1998), Section XI, Division 1, Article A-3000

Benthem, J.P. and Koiter, W.T. (1972) In : Methods of analysis of crack problems (Sih, G.C. Ed.),Vol. 3, pp. $131-178$

Chapuliot, S. et al (1998) Proc. ASME PVP Conference, Vol. 365, pp. 95-106

Chapuliot, S. et al (1999) Proc. ASME PVP Conference, Vol. 388, pp. 3-12

Green, A.E. and Sneddon, I.N. (1950) Proc. Camb. Phil. Soc., Vol. 46, pp. 159-163

Guozhong, C. Kangda, Z. and Dongdi, W. (1996) Engineering Fracture Mechanics, Vol. 54, No. 4, pp. 579-588

Isida, M. (1956) Trans. JSME, Vol. 22, pp. 809-814

Isida, M. (1966) Trans. ASME, Ser. E, J. Appl. Mech., Vol. 33, No. 3, pp. 674-675

Isida, M. (1976) In : Fracture mechanics and strength of materials 2, p. 128

Isida, M. and Noguchi, H. (1984) Engineering Fracture Mechanics, Vol. 20, No. 3, pp. 387-408

Irwin, G.R. (1962) Trans. ASME, Ser. E, J. Appl. Mech., Vol. 29, pp. 651-654

Kassir, M.K. and Sih, G.C. (1975) Mechanics of fracture 2. Three-dimensional crack problems. Noordhoff International Publishing

Nisitani, H. and Murakami, Y. (1974) Int. Journal of Fracture, Vol. 10, No. 3, pp. 353-368

RSE-M Code, 1997 Edition and 2000 Addenda, Appendix 5.4-II, AFCEN, Paris

Shah, R.C. and Kobayashi, A.S. (1971) Engineering Fracture Mechanics, Vol. 3, pp. 71-96

Shah, R.C. and Kobayashi, A.S. (1971) Proc. 1971 National Symposium on Fracture Mechanics, Part 1, ASTM STP 513, pp. 3-21

Shah, R.C. and Kobayashi, A.S. (1973) Int. Journal of Fracture, Vol. 9, No. 2, pp. 133-146

Shah, R.C. and Kobayashi, A.S. (1974) Trans. ASME, J. of Pressure Vessel Technology, pp. 47-54

Shiratori, M., Ogawa, T. and Nishijima, A. (1992) Trans. JSME, Vol. 58, No. 545, pp. 48-52

Stress intensity factors handbook in 3 volumes (1992) (Murakami, Y. Ed.), Pergamon Press, Oxford

Wadier, Y. and Malak, O. (1989) Proc. SMIRT 10, Vol. G, pp. 13-18

Wu, K.C. (2000) Int. Journal of Solids and Structures, Vol. 37, pp. 4841-4857 
TABLE 1 - Values of the influence coefficient $i_{0}$ at point $A$.

\begin{tabular}{|c|c|c|c|c|c|c|c|c|c|c|c|c|}
\hline & & & & & & & $a / d$ & & & & & \\
\hline$a / c$ & $2 a / t$ & 0.05 & 0.1 & 0.2 & 0.3 & 0.4 & 0.5 & 0.6 & 0.7 & 0.8 & 0.85 & 0.9 \\
\hline \multirow{6}{*}{1} & 0.05 & 0.6353 & 0.6354 & 0.6359 & 0.6373 & 0.6404 & 0.6459 & 0.6564 & 0.6757 & 0.7119 & 0.7423 & 0.7921 \\
\hline & 0.1 & & 0.6356 & 0.6362 & 0.6377 & 0.6406 & 0.6465 & 0.6572 & 0.6760 & 0.7116 & 0.7427 & 0.7926 \\
\hline & 0.2 & & & 0.6368 & 0.6385 & 0.6418 & 0.6480 & 0.6587 & 0.6787 & 0.7138 & 0.7452 & 0.7963 \\
\hline & 0.3 & & & & 0.6391 & 0.6422 & 0.6488 & 0.6602 & 0.6795 & 0.7163 & 0.7476 & 0.8005 \\
\hline & 0.4 & & & & & 0.6435 & 0.6499 & 0.6623 & 0.6819 & 0.7191 & 0.7508 & 0.8053 \\
\hline & 0.5 & & & & & & 0.6511 & 0.6631 & 0.6841 & 0.7230 & 0.7554 & 0.8119 \\
\hline \multirow{6}{*}{0.5} & 0.05 & 0.8245 & 0.8248 & 0.8262 & 0.8303 & 0.8391 & 0.8542 & 0.8807 & 0.9244 & 1.0007 & 1.0616 & 1.1594 \\
\hline & 0.1 & & 0.8253 & 0.8269 & 0.8312 & 0.8397 & 0.8554 & 0.8820 & 0.9246 & 1.0005 & 1.0632 & 1.1614 \\
\hline & 0.2 & & & 0.8284 & 0.8327 & 0.8422 & 0.8587 & 0.8853 & 0.9308 & 1.0065 & 1.0692 & 1.1721 \\
\hline & 0.3 & & & & 0.8352 & 0.8443 & 0.8618 & 0.8906 & 0.9357 & 1.0154 & 1.0786 & 1.1841 \\
\hline & 0.4 & & & & & 0.8499 & 0.8665 & 0.8977 & 0.9441 & 1.0248 & 1.0899 & 1.1991 \\
\hline & 0.5 & & & & & & 0.8725 & 0.9018 & 0.9520 & 1.0325 & 1.1031 & 1.2202 \\
\hline \multirow{6}{*}{0.25} & 0.05 & 0.9317 & 0.9323 & 0.9357 & 0.9443 & 0.9605 & 0.9872 & 1.0291 & 1.0952 & 1.2067 & 1.2964 & 1.4401 \\
\hline & 0.1 & & 0.9331 & 0.9366 & 0.9455 & 0.9618 & 0.9890 & 1.0315 & 1.0954 & 1.2080 & 1.3013 & 1.4447 \\
\hline & 0.2 & & & 0.9402 & 0.9493 & 0.9678 & 0.9970 & 1.0406 & 1.1105 & 1.2202 & 1.3145 & 1.4679 \\
\hline & 0.3 & & & & 0.9558 & 0.9724 & 1.0045 & 1.0525 & 1.1235 & 1.2444 & 1.3422 & 1.4991 \\
\hline & 0.4 & & & & & 0.9830 & 1.0122 & 1.0651 & 1.1400 & 1.2702 & 1.3746 & 1.5414 \\
\hline & 0.5 & & & & & & 1.0257 & 1.0746 & 1.1575 & 1.2951 & 1.4077 & 1.5851 \\
\hline \multirow{6}{*}{0.125} & 0.05 & 0.9771 & 0.9781 & 0.9839 & 0.9968 & 1.0189 & 1.0525 & 1.1034 & 1.1829 & 1.3165 & 1.4254 & 1.5996 \\
\hline & 0.1 & & 0.9796 & 0.9864 & 1.0004 & 1.0231 & 1.0578 & 1.1101 & 1.1881 & 1.3225 & 1.4348 & 1.6112 \\
\hline & 0.2 & & & 0.9907 & 1.0046 & 1.0307 & 1.0689 & 1.1238 & 1.2098 & 1.3539 & 1.4711 & 1.6589 \\
\hline & 0.3 & & & & 1.0135 & 1.0375 & 1.0809 & 1.1438 & 1.2346 & 1.4001 & 1.5259 & 1.7280 \\
\hline & 0.4 & & & & & 1.0546 & 1.0952 & 1.1674 & 1.2677 & 1.4424 & 1.5808 & 1.8023 \\
\hline & 0.5 & & & & & & 1.1117 & 1.1788 & 1.2919 & 1.4813 & 1.6351 & 1.8780 \\
\hline \multirow{6}{*}{0} & 0.05 & 1.0012 & 1.0045 & 1.0152 & 1.0323 & 1.0586 & 1.0979 & 1.1562 & 1.2471 & 1.4009 & 1.5235 & 1.7187 \\
\hline & 0.1 & & 1.0059 & 1.0195 & 1.0410 & 1.0708 & 1.1132 & 1.1752 & 1.2694 & 1.4266 & 1.5567 & 1.7592 \\
\hline & 0.2 & & & 1.0245 & 1.0502 & 1.0923 & 1.1478 & 1.2237 & 1.3342 & 1.5157 & 1.6642 & 1.8993 \\
\hline & 0.3 & & & & 1.0576 & 1.0991 & 1.1689 & 1.2648 & 1.4017 & 1.6210 & 1.7967 & 2.0771 \\
\hline & 0.4 & & & & & 1.1092 & 1.1740 & 1.2849 & 1.4495 & 1.7141 & 1.9267 & 2.2674 \\
\hline & 0.5 & & & & & & 1.1864 & 1.2887 & 1.4698 & 1.7747 & 2.0242 & 2.4247 \\
\hline
\end{tabular}


TABLE 2 - Values of the influence coefficient $i_{0}$ at point $B$.

\begin{tabular}{|c|c|c|c|c|c|c|c|c|c|c|c|c|}
\hline & & & & & & & $a / d$ & & & & & \\
\hline $\mathbf{a} / \mathbf{c}$ & $2 a / t$ & 0.05 & 0.1 & 0.2 & 0.3 & 0.4 & 0.5 & 0.6 & 0.7 & 0.8 & 0.85 & 0.9 \\
\hline \multirow{6}{*}{1} & 0.05 & 0.6353 & 0.6354 & 0.6358 & 0.6365 & 0.6376 & 0.6391 & 0.6414 & 0.6443 & 0.6478 & 0.6498 & 0.6528 \\
\hline & 0.1 & & 0.6356 & 0.6360 & 0.6368 & 0.6380 & 0.6397 & 0.6418 & 0.6446 & 0.6481 & 0.6501 & 0.6533 \\
\hline & 0.2 & & & 0.6368 & 0.6374 & 0.6389 & 0.6408 & 0.6432 & 0.6461 & 0.6495 & 0.6516 & 0.6548 \\
\hline & 0.3 & & & & 0.6391 & 0.6394 & 0.6412 & 0.6438 & 0.6470 & 0.6505 & 0.6527 & 0.6566 \\
\hline & 0.4 & & & & & 0.6435 & 0.6428 & 0.6449 & 0.6482 & 0.6514 & 0.6535 & 0.6578 \\
\hline & 0.5 & & & & & & 0.6511 & 0.6482 & 0.6497 & 0.6533 & 0.6550 & 0.6597 \\
\hline \multirow{6}{*}{0.5} & 0.05 & 0.8245 & 0.8247 & 0.8259 & 0.8283 & 0.8321 & 0.8372 & 0.8450 & 0.8538 & 0.8668 & 0.8739 & 0.8838 \\
\hline & 0.1 & & 0.8253 & 0.8264 & 0.8289 & 0.8329 & 0.8385 & 0.8458 & 0.8549 & 0.8675 & 0.8750 & 0.8851 \\
\hline & 0.2 & & & 0.8284 & 0.8302 & 0.8347 & 0.8409 & 0.8489 & 0.8586 & 0.8714 & 0.8787 & 0.8902 \\
\hline & 0.3 & & & & 0.8352 & 0.8366 & 0.8428 & 0.8514 & 0.8623 & 0.8758 & 0.8835 & 0.8959 \\
\hline & 0.4 & & & & & 0.8499 & 0.8491 & 0.8563 & 0.8675 & 0.8795 & 0.8872 & 0.9006 \\
\hline & 0.5 & & & & & & 0.8725 & 0.8666 & 0.8735 & 0.8828 & 0.8916 & 0.9085 \\
\hline \multirow{6}{*}{0.25} & 0.05 & 0.9317 & 0.9322 & 0.9348 & 0.9399 & 0.9479 & 0.9593 & 0.9744 & 0.9929 & 1.0162 & 1.0308 & 1.0499 \\
\hline & 0.1 & & 0.9331 & 0.9355 & 0.9410 & 0.9495 & 0.9611 & 0.9756 & 0.9933 & 1.0184 & 1.0344 & 1.0532 \\
\hline & 0.2 & & & 0.9402 & 0.9444 & 0.9541 & 0.9671 & 0.9835 & 1.0030 & 1.0261 & 1.0421 & 1.0647 \\
\hline & 0.3 & & & & 0.9558 & 0.9590 & 0.9721 & 0.9899 & 1.0121 & 1.0391 & 1.0566 & 1.0792 \\
\hline & 0.4 & & & & & 0.9830 & 0.9827 & 0.9978 & 1.0209 & 1.0500 & 1.0694 & 1.0943 \\
\hline & 0.5 & & & & & & 1.0257 & 1.0176 & 1.0334 & 1.0614 & 1.0818 & 1.1083 \\
\hline \multirow{6}{*}{0.125} & 0.05 & 0.9771 & 0.9779 & 0.9824 & 0.9907 & 1.0028 & 1.0189 & 1.0388 & 1.0637 & 1.0956 & 1.1161 & 1.1428 \\
\hline & 0.1 & & 0.9796 & 0.9847 & 0.9940 & 1.0071 & 1.0235 & 1.0436 & 1.0685 & 1.1014 & 1.1228 & 1.1505 \\
\hline & 0.2 & & & 0.9907 & 0.9974 & 1.0123 & 1.0312 & 1.0543 & 1.0815 & 1.1216 & 1.1449 & 1.1751 \\
\hline & 0.3 & & & & 1.0135 & 1.0189 & 1.0386 & 1.0647 & 1.0970 & 1.1459 & 1.1728 & 1.2075 \\
\hline & 0.4 & & & & & 1.0546 & 1.0555 & 1.0790 & 1.1145 & 1.1614 & 1.1922 & 1.2321 \\
\hline & 0.5 & & & & & & 1.1117 & 1.1028 & 1.1286 & 1.1752 & 1.2085 & 1.2523 \\
\hline \multirow{6}{*}{0} & 0.05 & 1.0012 & 1.0040 & 1.0128 & 1.0247 & 1.0403 & 1.0599 & 1.0841 & 1.1144 & 1.1549 & 1.1803 & 1.2125 \\
\hline & 0.1 & & 1.0059 & 1.0163 & 1.0320 & 1.0505 & 1.0726 & 1.0995 & 1.1326 & 1.1752 & 1.2028 & 1.2379 \\
\hline & 0.2 & & & 1.0245 & 1.0376 & 1.0643 & 1.0957 & 1.1321 & 1.1755 & 1.2312 & 1.2673 & 1.3134 \\
\hline & 0.3 & & & & 1.0576 & 1.0683 & 1.1037 & 1.1503 & 1.2076 & 1.2815 & 1.3292 & 1.3909 \\
\hline & 0.4 & & & & & 1.1092 & 1.1124 & 1.1546 & 1.2193 & 1.3095 & 1.3702 & 1.4507 \\
\hline & 0.5 & & & & & & 1.1864 & 1.1760 & 1.2245 & 1.3161 & 1.3839 & 1.4772 \\
\hline
\end{tabular}

TABLE 3 - Values of the influence coefficient $i_{0}$ at point $C$.

\begin{tabular}{|c|c|c|c|c|c|c|c|c|c|c|c|c|}
\hline & & & & & & & $\mathbf{a} / \mathbf{d}$ & & & & & \\
\hline $\mathrm{a} / \mathrm{c}$ & $2 \mathrm{a} / \mathrm{t}$ & 0.05 & 0.1 & 0.2 & 0.3 & 0.4 & 0.5 & 0.6 & 0.7 & 0.8 & 0.85 & 0.9 \\
\hline \multirow{6}{*}{1} & 0.05 & 0.6358 & 0.6359 & 0.6363 & 0.6374 & 0.6391 & 0.6415 & 0.6455 & 0.6510 & 0.6583 & 0.6627 & 0.6687 \\
\hline & 0.1 & & 0.6361 & 0.6365 & 0.6375 & 0.6393 & 0.6421 & 0.6460 & 0.6513 & 0.6585 & 0.6630 & 0.6691 \\
\hline & 0.2 & & & 0.6372 & 0.6382 & 0.6403 & 0.6433 & 0.6475 & 0.6533 & 0.6602 & 0.6649 & 0.6711 \\
\hline & 0.3 & & & & 0.6393 & 0.6408 & 0.6439 & 0.6485 & 0.6545 & 0.6618 & 0.6666 & 0.6737 \\
\hline & 0.4 & & & & & 0.6430 & 0.6451 & 0.6496 & 0.6561 & 0.6634 & 0.6682 & 0.6761 \\
\hline & 0.5 & & & & & & 0.6487 & 0.6516 & 0.6570 & 0.6659 & 0.6708 & 0.6796 \\
\hline \multirow{6}{*}{0.5} & 0.05 & 0.5831 & 0.5832 & 0.5840 & 0.5859 & 0.5888 & 0.5928 & 0.5991 & 0.6064 & 0.6155 & 0.6210 & 0.6292 \\
\hline & 0.1 & & 0.5834 & 0.5843 & 0.5863 & 0.5895 & 0.5939 & 0.5997 & 0.6069 & 0.6160 & 0.6217 & 0.6300 \\
\hline & 0.2 & & & 0.5854 & 0.5872 & 0.5908 & 0.5957 & 0.6019 & 0.6098 & 0.6191 & 0.6247 & 0.6343 \\
\hline & 0.3 & & & & 0.5895 & 0.5923 & 0.5974 & 0.6044 & 0.6130 & 0.6232 & 0.6290 & 0.6394 \\
\hline & 0.4 & & & & & 0.5972 & 0.6006 & 0.6074 & 0.6168 & 0.6268 & 0.6331 & 0.6443 \\
\hline & 0.5 & & & & & & 0.6071 & 0.6113 & 0.6196 & 0.6294 & 0.6374 & 0.6515 \\
\hline \multirow{6}{*}{0.25} & 0.05 & 0.4663 & 0.4666 & 0.4677 & 0.4696 & 0.4723 & 0.4758 & 0.4799 & 0.4847 & 0.4906 & 0.4951 & 0.5033 \\
\hline & 0.1 & & 0.4669 & 0.4681 & 0.4702 & 0.4730 & 0.4765 & 0.4807 & 0.4853 & 0.4914 & 0.4970 & 0.5043 \\
\hline & 0.2 & & & 0.4697 & 0.4719 & 0.4753 & 0.4795 & 0.4844 & 0.4902 & 0.4954 & 0.5006 & 0.5099 \\
\hline & 0.3 & & & & 0.4746 & 0.4773 & 0.4819 & 0.4878 & 0.4947 & 0.5020 & 0.5080 & 0.5172 \\
\hline & 0.4 & & & & & 0.4813 & 0.4844 & 0.4904 & 0.4984 & 0.5075 & 0.5144 & 0.5250 \\
\hline & 0.5 & & & & & & 0.4900 & 0.4938 & 0.5014 & 0.5118 & 0.5198 & 0.5316 \\
\hline \multirow{6}{*}{0.125} & 0.05 & 0.3480 & 0.3483 & 0.3492 & 0.3504 & 0.3520 & 0.3538 & 0.3560 & 0.3586 & 0.3615 & 0.3665 & 0.3754 \\
\hline & 0.1 & & 0.3484 & 0.3502 & 0.3519 & 0.3539 & 0.3559 & 0.3581 & 0.3601 & 0.3627 & 0.3679 & 0.3759 \\
\hline & 0.2 & & & 0.3513 & 0.3530 & 0.3555 & 0.3583 & 0.3613 & 0.3647 & 0.3680 & 0.3736 & 0.3841 \\
\hline & 0.3 & & & & 0.3544 & 0.3568 & 0.3599 & 0.3640 & 0.3684 & 0.3734 & 0.3796 & 0.3905 \\
\hline & 0.4 & & & & & 0.3587 & 0.3610 & 0.3660 & 0.3709 & 0.3767 & 0.3836 & 0.3959 \\
\hline & 0.5 & & & & & & 0.3637 & 0.3667 & 0.3722 & 0.3784 & 0.3860 & 0.3995 \\
\hline
\end{tabular}


TABLE 4 - Values of the influence coefficient $i_{1}$ at point $A$.

\begin{tabular}{|c|c|c|c|c|c|c|c|c|c|c|c|c|}
\hline & & & & & & & $a / d$ & & & & & \\
\hline$a / c$ & $2 a / t$ & 0.05 & 0.1 & 0.2 & 0.3 & 0.4 & 0.5 & 0.6 & 0.7 & 0.8 & 0.85 & 0.9 \\
\hline \multirow{6}{*}{1} & 0.05 & -0.4231 & -0.4232 & -0.4232 & -0.4235 & -0.4240 & -0.4248 & -0.4273 & -0.4334 & -0.4482 & -0.4630 & -0.4902 \\
\hline & 0.1 & & -0.4232 & -0.4232 & -0.4234 & -0.4238 & -0.4248 & -0.4275 & -0.4334 & -0.4479 & -0.4630 & -0.4903 \\
\hline & 0.2 & & & -0.4232 & -0.4234 & -0.4238 & -0.4249 & -0.4272 & -0.4337 & -0.4480 & -0.4632 & -0.4909 \\
\hline & 0.3 & & & & -0.4234 & -0.4238 & -0.4249 & -0.4275 & -0.4332 & -0.4483 & -0.4632 & -0.4912 \\
\hline & 0.4 & & & & & -0.4238 & -0.4251 & -0.4281 & -0.4334 & -0.4487 & -0.4637 & -0.4920 \\
\hline & 0.5 & & & & & & -0.4251 & -0.4282 & -0.4344 & -0.4494 & -0.4647 & -0.4931 \\
\hline \multirow{6}{*}{0.5} & 0.05 & -0.4734 & -0.4734 & -0.4734 & -0.4741 & -0.4753 & -0.4771 & -0.4824 & -0.4937 & -0.5191 & -0.5431 & -0.5860 \\
\hline & 0.1 & & -0.4735 & -0.4736 & -0.4739 & -0.4748 & -0.4772 & -0.4826 & -0.4931 & -0.5185 & -0.5431 & -0.5861 \\
\hline & 0.2 & & & -0.4737 & -0.4740 & -0.4750 & -0.4774 & -0.4822 & -0.4941 & -0.5188 & -0.5434 & -0.5879 \\
\hline & 0.3 & & & & -0.4739 & -0.4752 & -0.4775 & -0.4829 & -0.4937 & -0.5195 & -0.5439 & -0.5886 \\
\hline & 0.4 & & & & & -0.4749 & -0.4778 & -0.4839 & -0.4942 & -0.5205 & -0.5453 & -0.5906 \\
\hline & 0.5 & & & & & & -0.4776 & -0.4843 & -0.4963 & -0.5222 & -0.5477 & -0.5935 \\
\hline \multirow{6}{*}{0.25} & 0.05 & -0.4918 & -0.4919 & -0.4920 & -0.4926 & -0.4943 & -0.4977 & -0.5052 & -0.5204 & -0.5529 & -0.5838 & -0.6382 \\
\hline & 0.1 & & -0.4921 & -0.4922 & -0.4928 & -0.4943 & -0.4979 & -0.5056 & -0.5197 & -0.5523 & -0.5841 & -0.6382 \\
\hline & 0.2 & & & -0.4922 & -0.4928 & -0.4946 & -0.4982 & -0.5053 & -0.5213 & -0.5528 & -0.5846 & -0.6405 \\
\hline & 0.3 & & & & -0.4927 & -0.4947 & -0.4987 & -0.5064 & -0.5210 & -0.5544 & -0.5862 & -0.6426 \\
\hline & 0.4 & & & & & -0.4944 & -0.4992 & -0.5082 & -0.5224 & -0.5570 & -0.5895 & -0.6474 \\
\hline & 0.5 & & & & & & -0.4987 & -0.5088 & -0.5258 & -0.5607 & -0.5945 & -0.6537 \\
\hline \multirow{6}{*}{0.125} & 0.05 & -0.4975 & -0.4976 & -0.4978 & -0.4987 & -0.5008 & -0.5049 & -0.5134 & -0.5305 & -0.5670 & -0.6018 & -0.6630 \\
\hline & 0.1 & & -0.4976 & -0.4979 & -0.4986 & -0.5006 & -0.5050 & -0.5139 & -0.5299 & -0.5665 & -0.6021 & -0.6634 \\
\hline & 0.2 & & & -0.4978 & -0.4989 & -0.5010 & -0.5054 & -0.5138 & -0.5320 & -0.5679 & -0.6037 & -0.6670 \\
\hline & 0.3 & & & & -0.4985 & -0.5013 & -0.5063 & -0.5155 & -0.5323 & -0.5710 & -0.6073 & -0.6718 \\
\hline & 0.4 & & & & & -0.5009 & -0.5072 & -0.5183 & -0.5352 & -0.5758 & -0.6136 & -0.6807 \\
\hline & 0.5 & & & & & & -0.5066 & -0.5199 & -0.5405 & -0.5825 & -0.6224 & -0.6926 \\
\hline \multirow{6}{*}{0} & 0.05 & -0.4998 & -0.4999 & -0.5002 & -0.5011 & -0.5034 & -0.5081 & -0.5174 & -0.5361 & -0.5760 & -0.6129 & -0.6775 \\
\hline & 0.1 & & -0.4998 & -0.5003 & -0.5013 & -0.5036 & -0.5085 & -0.5180 & -0.5368 & -0.5764 & -0.6146 & -0.6800 \\
\hline & 0.2 & & & -0.5002 & -0.5020 & -0.5049 & -0.5102 & -0.5204 & -0.5401 & -0.5817 & -0.6217 & -0.6917 \\
\hline & 0.3 & & & & -0.5015 & -0.5062 & -0.5130 & -0.5246 & -0.5463 & -0.5914 & -0.6340 & -0.7096 \\
\hline & 0.4 & & & & & -0.5054 & -0.5153 & -0.5295 & -0.5545 & -0.6048 & -0.6522 & -0.7365 \\
\hline & 0.5 & & & & & & -0.5143 & -0.5334 & -0.5630 & -0.6202 & -0.6737 & -0.7680 \\
\hline
\end{tabular}


TABLE 5 - Values of the influence coefficient $i_{1}$ at point $B$.

\begin{tabular}{|c|c|c|c|c|c|c|c|c|c|c|c|c|}
\hline & & & & & & & $a / d$ & & & & & \\
\hline $\mathbf{a} / \mathbf{c}$ & $2 a / t$ & 0.05 & 0.1 & 0.2 & 0.3 & 0.4 & 0.5 & 0.6 & 0.7 & 0.8 & 0.85 & 0.9 \\
\hline \multirow{6}{*}{1} & 0.05 & 0.4231 & 0.4232 & 0.4232 & 0.4232 & 0.4231 & 0.4230 & 0.4228 & 0.4225 & 0.4221 & 0.4218 & 0.4215 \\
\hline & 0.1 & & 0.4232 & 0.4232 & 0.4231 & 0.4231 & 0.4230 & 0.4227 & 0.4225 & 0.4221 & 0.4218 & 0.4215 \\
\hline & 0.2 & & & 0.4232 & 0.4232 & 0.4231 & 0.4230 & 0.4228 & 0.4224 & 0.4221 & 0.4218 & 0.4215 \\
\hline & 0.3 & & & & 0.4234 & 0.4231 & 0.4230 & 0.4227 & 0.4224 & 0.4220 & 0.4217 & 0.4214 \\
\hline & 0.4 & & & & & 0.4238 & 0.4233 & 0.4229 & 0.4224 & 0.4220 & 0.4217 & 0.4214 \\
\hline & 0.5 & & & & & & 0.4251 & 0.4238 & 0.4231 & 0.4223 & 0.4220 & 0.4217 \\
\hline \multirow{6}{*}{0.5} & 0.05 & 0.4734 & 0.4734 & 0.4734 & 0.4733 & 0.4730 & 0.4727 & 0.4722 & 0.4709 & 0.4698 & 0.4689 & 0.4681 \\
\hline & 0.1 & & 0.4735 & 0.4735 & 0.4734 & 0.4732 & 0.4728 & 0.4721 & 0.4710 & 0.4697 & 0.4689 & 0.4681 \\
\hline & 0.2 & & & $\begin{array}{l}0.4737 \\
\end{array}$ & 0.4736 & 0.4733 & 0.4729 & 0.4722 & 0.4710 & 0.4696 & 0.4687 & 0.4680 \\
\hline & 0.3 & & & & 0.4739 & 0.4733 & 0.4728 & 0.4720 & 0.4710 & 0.4694 & 0.4684 & 0.4674 \\
\hline & 0.4 & & & & & 0.4749 & 0.4734 & 0.4723 & 0.4710 & 0.4693 & 0.4682 & 0.4672 \\
\hline & 0.5 & & & & & & 0.4776 & 0.4743 & 0.4722 & 0.4702 & 0.4688 & 0.4677 \\
\hline \multirow{6}{*}{0.25} & 0.05 & 0.4918 & 0.4918 & 0.4918 & 0.4916 & 0.4911 & 0.4903 & 0.4894 & 0.4877 & 0.4849 & 0.4835 & 0.4819 \\
\hline & 0.1 & & 0.4921 & 0.4920 & 0.4918 & 0.4914 & 0.4906 & 0.4893 & 0.4873 & 0.4848 & 0.4835 & 0.4819 \\
\hline & 0.2 & & & 0.4922 & 0.4918 & 0.4913 & 0.4905 & 0.4892 & 0.4873 & 0.4845 & 0.4829 & 0.4812 \\
\hline & 0.3 & & & & 0.4927 & 0.4916 & 0.4905 & 0.4890 & 0.4870 & 0.4838 & 0.4821 & 0.4803 \\
\hline & 0.4 & & & & & 0.4944 & 0.4917 & 0.4895 & 0.4870 & 0.4834 & 0.4814 & 0.4791 \\
\hline & 0.5 & & & & & & 0.4987 & 0.4930 & 0.4890 & 0.4844 & 0.4820 & 0.4793 \\
\hline \multirow{6}{*}{0.125} & 0.05 & 0.4975 & 0.4975 & 0.4975 & 0.4972 & 0.4966 & 0.4956 & 0.4940 & 0.4917 & 0.4879 & 0.4857 & 0.4830 \\
\hline & 0.1 & & 0.4976 & 0.4975 & 0.4971 & 0.4965 & 0.4955 & 0.4939 & 0.4913 & 0.4879 & 0.4855 & 0.4828 \\
\hline & 0.2 & & & 0.4978 & 0.4971 & 0.4964 & 0.4953 & 0.4936 & 0.4910 & 0.4870 & 0.4843 & 0.4811 \\
\hline & 0.3 & & & & 0.4985 & 0.4968 & 0.4952 & 0.4932 & 0.4904 & 0.4856 & 0.4826 & 0.4789 \\
\hline & 0.4 & & & & & 0.5009 & 0.4969 & 0.4937 & 0.4900 & 0.4846 & 0.4811 & 0.4767 \\
\hline & 0.5 & & & & & & 0.5066 & 0.4989 & 0.4930 & 0.4858 & 0.4815 & 0.4762 \\
\hline \multirow{6}{*}{0} & 0.05 & 0.4998 & 0.4997 & 0.4996 & 0.4991 & 0.4983 & 0.4971 & 0.4951 & 0.4918 & 0.4866 & 0.4827 & 0.4767 \\
\hline & 0.1 & & 0.4998 & 0.4995 & 0.4990 & 0.4981 & 0.4968 & 0.4947 & 0.4915 & 0.4861 & 0.4816 & 0.4753 \\
\hline & 0.2 & & & 0.5002 & 0.4988 & 0.4974 & 0.4957 & 0.4931 & 0.4893 & 0.4829 & 0.4778 & 0.4699 \\
\hline & 0.3 & & & & 0.5015 & 0.4981 & 0.4951 & 0.4915 & 0.4865 & 0.4784 & 0.4720 & 0.4619 \\
\hline & 0.4 & & & & & 0.5054 & 0.4984 & 0.4926 & 0.4856 & 0.4750 & 0.4668 & 0.4542 \\
\hline & 0.5 & & & & & & 0.5143 & 0.5014 & 0.4905 & 0.4765 & 0.4663 & 0.4508 \\
\hline
\end{tabular}

TABLE 6 - Values of the influence coefficient $i_{1}$ at point $C$.

\begin{tabular}{|c|c|c|c|c|c|c|c|c|c|c|c|c|}
\hline & & & & & & & $a / d$ & & & & & \\
\hline $\mathbf{a} / \mathbf{c}$ & $2 \mathrm{a} / \mathrm{t}$ & 0.05 & 0.1 & 0.2 & 0.3 & 0.4 & 0.5 & 0.6 & 0.7 & 0.8 & 0.85 & 0.9 \\
\hline \multirow{6}{*}{1} & 0.05 & -0.0001 & -0.0001 & -0.0002 & -0.0002 & -0.0004 & -0.0007 & -0.0012 & -0.0020 & -0.0032 & -0.0041 & -0.0051 \\
\hline & 0.1 & & -0.0001 & -0.0002 & -0.0002 & -0.0004 & -0.0007 & -0.0012 & -0.0020 & -0.0032 & -0.0041 & -0.0051 \\
\hline & 0.2 & & & -0.0001 & -0.0002 & -0.0004 & -0.0007 & -0.0012 & -0.0021 & -0.0033 & -0.0042 & -0.0053 \\
\hline & 0.3 & & & & -0.0001 & -0.0004 & -0.0007 & -0.0013 & -0.0021 & -0.0034 & -0.0043 & -0.0055 \\
\hline & 0.4 & & & & & -0.0001 & -0.0007 & -0.0014 & -0.0023 & -0.0036 & -0.0045 & -0.0058 \\
\hline & 0.5 & & & & & & -0.0001 & -0.0012 & -0.0023 & -0.0038 & -0.0048 & -0.0062 \\
\hline \multirow{6}{*}{0.5} & 0.05 & -0.0001 & -0.0001 & -0.0001 & -0.0002 & -0.0004 & -0.0008 & -0.0013 & -0.0020 & -0.0032 & -0.0039 & -0.0049 \\
\hline & 0.1 & & -0.0001 & -0.0001 & -0.0002 & -0.0004 & -0.0008 & -0.0013 & -0.0020 & -0.0032 & -0.0039 & -0.0050 \\
\hline & 0.2 & & & -0.0001 & -0.0002 & -0.0005 & -0.0008 & -0.0014 & -0.0022 & -0.0033 & -0.0041 & -0.0052 \\
\hline & 0.3 & & & & -0.0001 & -0.0005 & -0.0009 & -0.0015 & -0.0024 & -0.0036 & -0.0044 & -0.0056 \\
\hline & 0.4 & & & & & -0.0001 & -0.0008 & -0.0016 & -0.0026 & -0.0039 & -0.0048 & -0.0061 \\
\hline & 0.5 & & & & & & -0.0001 & -0.0014 & -0.0026 & -0.0042 & -0.0053 & -0.0068 \\
\hline \multirow{6}{*}{0.25} & 0.05 & -0.0001 & -0.0001 & -0.0001 & -0.0002 & -0.0003 & -0.0004 & -0.0007 & -0.0010 & -0.0015 & -0.0018 & -0.0023 \\
\hline & 0.1 & & -0.0001 & -0.0001 & -0.0002 & -0.0003 & -0.0005 & -0.0007 & -0.0010 & -0.0015 & -0.0019 & -0.0024 \\
\hline & 0.2 & & & -0.0001 & -0.0002 & -0.0003 & -0.0005 & -0.0008 & -0.0012 & -0.0017 & -0.0021 & -0.0027 \\
\hline & 0.3 & & & & -0.0001 & -0.0004 & -0.0007 & -0.0010 & -0.0015 & -0.0021 & -0.0026 & -0.0032 \\
\hline & 0.4 & & & & & -0.0001 & -0.0006 & -0.0011 & -0.0017 & -0.0026 & -0.0032 & -0.0040 \\
\hline & 0.5 & & & & & & -0.0001 & -0.0010 & -0.0019 & -0.0030 & -0.0038 & -0.0047 \\
\hline \multirow{6}{*}{0.125} & 0.05 & 0.0000 & 0.0000 & -0.0001 & -0.0001 & -0.0001 & -0.0002 & -0.0002 & -0.0003 & -0.0005 & -0.0006 & -0.0007 \\
\hline & 0.1 & & 0.0000 & -0.0001 & -0.0001 & -0.0001 & -0.0002 & -0.0003 & -0.0004 & -0.0005 & -0.0006 & -0.0008 \\
\hline & 0.2 & & & 0.0000 & -0.0001 & -0.0002 & -0.0003 & -0.0004 & -0.0005 & -0.0007 & -0.0009 & -0.0011 \\
\hline & 0.3 & & & & 0.0000 & -0.0002 & -0.0004 & -0.0005 & -0.0008 & -0.0011 & -0.0013 & -0.0017 \\
\hline & 0.4 & & & & & 0.0000 & -0.0004 & -0.0007 & -0.0010 & -0.0015 & -0.0018 & -0.0022 \\
\hline & 0.5 & & & & & & 0.0000 & -0.0006 & -0.0011 & -0.0018 & -0.0022 & -0.0028 \\
\hline
\end{tabular}


TABLE 7 - Values of the influence coefficient $i_{2}$ at point $A$.

\begin{tabular}{|c|c|c|c|c|c|c|c|c|c|c|c|c|}
\hline & & & & & & & $a / d$ & & & & & \\
\hline$a / c$ & $2 a / t$ & 0.05 & 0.1 & 0.2 & 0.3 & 0.4 & 0.5 & 0.6 & 0.7 & 0.8 & 0.85 & 0.9 \\
\hline \multirow{6}{*}{1} & 0.05 & 0.3805 & 0.3805 & 0.3806 & 0.3810 & 0.3818 & 0.3828 & 0.3854 & 0.3907 & 0.4026 & 0.4142 & 0.4358 \\
\hline & 0.1 & & 0.3805 & 0.3807 & 0.3810 & 0.3816 & 0.3829 & 0.3856 & 0.3908 & 0.4024 & 0.4143 & 0.4360 \\
\hline & 0.2 & & & 0.3808 & 0.3812 & 0.3819 & 0.3832 & 0.3858 & 0.3915 & 0.4029 & 0.4148 & 0.4370 \\
\hline & 0.3 & & & & 0.3813 & 0.3819 & 0.3834 & 0.3861 & 0.3912 & 0.4034 & 0.4152 & 0.4378 \\
\hline & 0.4 & & & & & 0.3822 & 0.3836 & 0.3867 & 0.3916 & 0.4039 & 0.4159 & 0.4388 \\
\hline & 0.5 & & & & & & 0.3839 & 0.3869 & 0.3924 & 0.4048 & 0.4169 & 0.4402 \\
\hline \multirow{6}{*}{0.5} & 0.05 & 0.4398 & 0.4399 & 0.4402 & 0.4414 & 0.4435 & 0.4463 & 0.4528 & 0.4644 & 0.4875 & 0.5084 & 0.5453 \\
\hline & 0.1 & & 0.4401 & 0.4404 & 0.4413 & 0.4431 & 0.4466 & 0.4531 & 0.4642 & 0.4873 & 0.5087 & 0.5458 \\
\hline & 0.2 & & & 0.4407 & 0.4417 & 0.4437 & 0.4473 & 0.4536 & 0.4658 & 0.4885 & 0.5099 & 0.5486 \\
\hline & 0.3 & & & & 0.4421 & 0.4441 & 0.4479 & 0.4547 & 0.4663 & 0.4903 & 0.5117 & 0.5510 \\
\hline & 0.4 & & & & & 0.4452 & 0.4489 & 0.4563 & 0.4678 & 0.4923 & 0.5141 & 0.5542 \\
\hline & 0.5 & & & & & & 0.4501 & 0.4572 & 0.4699 & 0.4940 & 0.5169 & 0.5586 \\
\hline \multirow{6}{*}{0.25} & 0.05 & 0.4737 & 0.4739 & 0.4745 & 0.4764 & 0.4800 & 0.4862 & 0.4968 & 0.5145 & 0.5477 & 0.5773 & 0.6281 \\
\hline & 0.1 & & 0.4741 & 0.4749 & 0.4768 & 0.4804 & 0.4867 & 0.4974 & 0.5144 & 0.5477 & 0.5782 & 0.6290 \\
\hline & 0.2 & & & 0.4756 & 0.4775 & 0.4816 & 0.4883 & 0.4989 & 0.5178 & 0.5502 & 0.5810 & 0.6343 \\
\hline & 0.3 & & & & 0.4789 & 0.4825 & 0.4899 & 0.5015 & 0.5198 & 0.5551 & 0.5866 & 0.6407 \\
\hline & 0.4 & & & & & 0.4847 & 0.4915 & 0.5044 & 0.5231 & 0.5605 & 0.5934 & 0.6498 \\
\hline & 0.5 & & & & & & 0.4943 & 0.5062 & 0.5273 & 0.5658 & 0.6005 & 0.6592 \\
\hline \multirow{6}{*}{0.125} & 0.05 & 0.4899 & 0.4901 & 0.4913 & 0.4943 & 0.4995 & 0.5078 & 0.5209 & 0.5427 & 0.5827 & 0.6183 & 0.6789 \\
\hline & 0.1 & & 0.4904 & 0.4918 & 0.4949 & 0.5003 & 0.5089 & 0.5225 & 0.5434 & 0.5837 & 0.6202 & 0.6813 \\
\hline & 0.2 & & & 0.4928 & 0.4958 & 0.5019 & 0.5111 & 0.5249 & 0.5484 & 0.5902 & 0.6277 & 0.6918 \\
\hline & 0.3 & & & & 0.4978 & 0.5034 & 0.5137 & 0.5293 & 0.5530 & 0.6000 & 0.6393 & 0.7065 \\
\hline & 0.4 & & & & & 0.5072 & 0.5168 & 0.5348 & 0.5599 & 0.6092 & 0.6514 & 0.7231 \\
\hline & 0.5 & & & & & & 0.5206 & 0.5373 & 0.5659 & 0.6180 & 0.6637 & 0.7403 \\
\hline \multirow{6}{*}{0} & 0.05 & 0.5000 & 0.5008 & 0.5035 & 0.5078 & 0.5145 & 0.5247 & 0.5404 & 0.5660 & 0.6128 & 0.6528 & 0.7207 \\
\hline & 0.1 & & 0.5012 & 0.5046 & 0.5100 & 0.5176 & 0.5286 & 0.5451 & 0.5716 & 0.6191 & 0.6614 & 0.7311 \\
\hline & 0.2 & & & 0.5059 & 0.5123 & 0.5230 & 0.5373 & 0.5573 & 0.5879 & 0.6417 & 0.6887 & 0.7675 \\
\hline & 0.3 & & & & 0.5142 & 0.5247 & 0.5425 & 0.5677 & 0.6049 & 0.6684 & 0.7224 & 0.8132 \\
\hline & 0.4 & & & & & 0.5272 & 0.5438 & 0.5727 & 0.6170 & 0.6921 & 0.7558 & 0.8627 \\
\hline & 0.5 & & & & & & 0.5469 & 0.5737 & 0.6221 & 0.7076 & 0.7809 & 0.9037 \\
\hline
\end{tabular}


TABLE 8 - Values of the influence coefficient $i_{2}$ at point $B$.

\begin{tabular}{|c|c|c|c|c|c|c|c|c|c|c|c|c|}
\hline & & & & & & & $a / d$ & & & & & \\
\hline $\mathbf{a} / \mathbf{c}$ & $2 a / t$ & 0.05 & 0.1 & 0.2 & 0.3 & 0.4 & 0.5 & 0.6 & 0.7 & 0.8 & 0.85 & 0.9 \\
\hline \multirow{6}{*}{1} & 0.05 & 0.3805 & 0.3805 & 0.3806 & 0.3807 & 0.3810 & 0.3813 & 0.3817 & 0.3824 & 0.3831 & 0.3835 & 0.3840 \\
\hline & 0.1 & & 0.3805 & 0.3806 & 0.3808 & 0.3810 & 0.3814 & 0.3818 & 0.3824 & 0.3831 & 0.3836 & 0.3841 \\
\hline & 0.2 & & & 0.3808 & 0.3809 & 0.3812 & 0.3816 & 0.3821 & 0.3827 & 0.3834 & 0.3839 & 0.3845 \\
\hline & 0.3 & & & & 0.3813 & 0.3813 & 0.3817 & 0.3822 & 0.3829 & 0.3836 & 0.3841 & 0.3847 \\
\hline & 0.4 & & & & & 0.3822 & 0.3820 & 0.3824 & 0.3831 & 0.3838 & 0.3843 & 0.3849 \\
\hline & 0.5 & & & & & & 0.3839 & 0.3832 & 0.3835 & 0.3842 & 0.3847 & 0.3853 \\
\hline \multirow{6}{*}{0.5} & 0.05 & 0.4398 & 0.4399 & 0.4401 & 0.4406 & 0.4414 & 0.4425 & 0.4442 & 0.4460 & 0.4489 & 0.4504 & 0.4524 \\
\hline & 0.1 & & 0.4401 & 0.4403 & 0.4408 & 0.4416 & 0.4428 & 0.4443 & 0.4461 & 0.4489 & 0.4507 & 0.4526 \\
\hline & 0.2 & & & 0.4407 & 0.4412 & 0.4420 & 0.4433 & 0.4450 & 0.4470 & 0.4498 & 0.4514 & 0.4534 \\
\hline & 0.3 & & & & 0.4421 & 0.4424 & 0.4437 & 0.4455 & 0.4478 & 0.4507 & 0.4524 & 0.4546 \\
\hline & 0.4 & & & & & 0.4452 & 0.4450 & 0.4465 & 0.4488 & 0.4514 & 0.4532 & 0.4554 \\
\hline & 0.5 & & & & & & 0.4501 & 0.4487 & 0.4501 & 0.4523 & 0.4542 & 0.4565 \\
\hline \multirow{6}{*}{0.25} & 0.05 & 0.4737 & 0.4738 & 0.4743 & 0.4754 & 0.4771 & 0.4796 & 0.4831 & 0.4872 & 0.4925 & 0.4963 & 0.5015 \\
\hline & 0.1 & & 0.4741 & 0.4746 & 0.4758 & 0.4776 & 0.4801 & 0.4832 & 0.4871 & 0.4928 & 0.4971 & 0.5022 \\
\hline & 0.2 & & & 0.4756 & 0.4765 & 0.4785 & 0.4813 & 0.4849 & 0.4892 & 0.4944 & 0.4985 & 0.5046 \\
\hline & 0.3 & & & & 0.4789 & 0.4796 & 0.4823 & 0.4862 & 0.4911 & 0.4971 & 0.5016 & 0.5076 \\
\hline & 0.4 & & & & & 0.4847 & 0.4846 & 0.4879 & 0.4929 & 0.4994 & 0.5042 & 0.5108 \\
\hline & 0.5 & & & & & & 0.4943 & 0.4923 & 0.4957 & 0.5019 & 0.5070 & 0.5140 \\
\hline \multirow{6}{*}{0.125} & 0.05 & 0.4899 & 0.4901 & 0.4910 & 0.4929 & 0.4956 & 0.4994 & 0.5040 & 0.5099 & 0.5176 & 0.5231 & 0.5307 \\
\hline & 0.1 & & 0.4904 & 0.4914 & 0.4934 & 0.4964 & 0.5003 & 0.5050 & 0.5107 & 0.5188 & 0.5245 & 0.5324 \\
\hline & 0.2 & & & 0.4928 & 0.4942 & 0.4976 & 0.5019 & 0.5072 & 0.5135 & 0.5230 & 0.5291 & 0.5374 \\
\hline & 0.3 & & & & 0.4978 & 0.4990 & 0.5034 & 0.5094 & 0.5168 & 0.5281 & 0.5350 & 0.5444 \\
\hline & 0.4 & & & & & 0.5072 & 0.5073 & 0.5126 & 0.5206 & 0.5316 & 0.5393 & 0.5498 \\
\hline & 0.5 & & & & & & 0.5206 & 0.5183 & 0.5241 & 0.5349 & 0.5432 & 0.5546 \\
\hline \multirow{6}{*}{0} & 0.05 & 0.5000 & 0.5007 & 0.5029 & 0.5059 & 0.5098 & 0.5147 & 0.5208 & 0.5283 & 0.5388 & 0.5456 & 0.5546 \\
\hline & 0.1 & & 0.5012 & 0.5038 & 0.5077 & 0.5124 & 0.5179 & 0.5247 & 0.5330 & 0.5440 & 0.5513 & 0.5612 \\
\hline & 0.2 & & & 0.5059 & 0.5091 & 0.5158 & 0.5237 & 0.5328 & 0.5438 & 0.5582 & 0.5679 & 0.5807 \\
\hline & 0.3 & & & & 0.5142 & 0.5168 & 0.5257 & 0.5374 & 0.5519 & 0.5710 & 0.5837 & 0.6006 \\
\hline & 0.4 & & & & & 0.5272 & 0.5279 & 0.5385 & 0.5549 & 0.5781 & 0.5942 & 0.6162 \\
\hline & 0.5 & & & & & & 0.5469 & 0.5441 & 0.5562 & 0.5798 & 0.5978 & 0.6231 \\
\hline
\end{tabular}

TABLE 9 - Values of the influence coefficient $i_{2}$ at point $C$.

\begin{tabular}{|c|c|c|c|c|c|c|c|c|c|c|c|c|}
\hline & & & & & & & $\mathbf{a} / \mathbf{d}$ & & & & & \\
\hline $\mathrm{a} / \mathrm{c}$ & $2 \mathrm{a} / \mathrm{t}$ & 0.05 & 0.1 & 0.2 & 0.3 & 0.4 & 0.5 & 0.6 & 0.7 & 0.8 & 0.85 & 0.9 \\
\hline \multirow{6}{*}{1} & 0.05 & 0.0417 & 0.0417 & 0.0418 & 0.0420 & 0.0423 & 0.0429 & 0.0437 & 0.0449 & 0.0465 & 0.0474 & 0.0487 \\
\hline & 0.1 & & 0.0418 & 0.0419 & 0.0421 & 0.0424 & 0.0430 & 0.0438 & 0.0449 & 0.0465 & 0.0475 & 0.0487 \\
\hline & 0.2 & & & 0.0420 & 0.0422 & 0.0426 & 0.0432 & 0.0442 & 0.0454 & 0.0468 & 0.0479 & 0.0491 \\
\hline & 0.3 & & & & 0.0424 & 0.0427 & 0.0434 & 0.0443 & 0.0456 & 0.0472 & 0.0482 & 0.0497 \\
\hline & 0.4 & & & & & 0.0432 & 0.0436 & 0.0446 & 0.0459 & 0.0475 & 0.0485 & 0.0502 \\
\hline & 0.5 & & & & & & 0.0443 & 0.0449 & 0.0462 & 0.0480 & 0.0490 & 0.0509 \\
\hline \multirow{6}{*}{0.5} & 0.05 & 0.0319 & 0.0319 & 0.0321 & 0.0324 & 0.0330 & 0.0339 & 0.0351 & 0.0366 & 0.0384 & 0.0396 & 0.0410 \\
\hline & 0.1 & & 0.0320 & 0.0322 & 0.0326 & 0.0332 & 0.0341 & 0.0353 & 0.0367 & 0.0385 & 0.0397 & 0.0412 \\
\hline & 0.2 & & & 0.0324 & 0.0327 & 0.0335 & 0.0345 & 0.0357 & 0.0372 & 0.0392 & 0.0403 & 0.0420 \\
\hline & 0.3 & & & & 0.0332 & 0.0337 & 0.0348 & 0.0362 & 0.0379 & 0.0400 & 0.0412 & 0.0431 \\
\hline & 0.4 & & & & & 0.0348 & 0.0354 & 0.0369 & 0.0387 & 0.0407 & 0.0420 & 0.0441 \\
\hline & 0.5 & & & & & & 0.0368 & 0.0376 & 0.0394 & 0.0413 & 0.0429 & 0.0456 \\
\hline \multirow{6}{*}{0.25} & 0.05 & 0.0148 & 0.0148 & 0.0151 & 0.0154 & 0.0160 & 0.0166 & 0.0173 & 0.0181 & 0.0191 & 0.0197 & 0.0206 \\
\hline & 0.1 & & 0.0149 & 0.0151 & 0.0155 & 0.0161 & 0.0167 & 0.0174 & 0.0182 & 0.0193 & 0.0200 & 0.0209 \\
\hline & 0.2 & & & 0.0154 & 0.0159 & 0.0165 & 0.0173 & 0.0182 & 0.0192 & 0.0201 & 0.0209 & 0.0221 \\
\hline & 0.3 & & & & 0.0164 & 0.0169 & 0.0178 & 0.0189 & 0.0201 & 0.0215 & 0.0224 & 0.0236 \\
\hline & 0.4 & & & & & 0.0177 & 0.0183 & 0.0194 & 0.0209 & 0.0226 & 0.0237 & 0.0252 \\
\hline & 0.5 & & & & & & 0.0193 & 0.0200 & 0.0215 & 0.0235 & 0.0248 & 0.0266 \\
\hline \multirow{6}{*}{0.125} & 0.05 & 0.0047 & 0.0047 & 0.0049 & 0.0051 & 0.0053 & 0.0055 & 0.0058 & 0.0060 & 0.0064 & 0.0066 & 0.0069 \\
\hline & 0.1 & & 0.0048 & 0.0050 & 0.0053 & 0.0056 & 0.0058 & 0.0061 & 0.0064 & 0.0067 & 0.0070 & 0.0073 \\
\hline & 0.2 & & & 0.0052 & 0.0055 & 0.0059 & 0.0063 & 0.0067 & 0.0072 & 0.0078 & 0.0082 & 0.0087 \\
\hline & 0.3 & & & & 0.0057 & 0.0061 & 0.0066 & 0.0073 & 0.0079 & 0.0089 & 0.0095 & 0.0103 \\
\hline & 0.4 & & & & & 0.0065 & 0.0069 & 0.0076 & 0.0085 & 0.0096 & 0.0103 & 0.0113 \\
\hline & 0.5 & & & & & & 0.0073 & 0.0077 & 0.0087 & 0.0099 & 0.0108 & 0.0120 \\
\hline
\end{tabular}


TABLE 10 - Values of the influence coefficient $i_{3}$ at point $A$.

\begin{tabular}{|c|c|c|c|c|c|c|c|c|c|c|c|c|}
\hline & & & & & & & $a / d$ & & & & & \\
\hline$a / c$ & $2 a / t$ & 0.05 & 0.1 & 0.2 & 0.3 & 0.4 & 0.5 & 0.6 & 0.7 & 0.8 & 0.85 & 0.9 \\
\hline \multirow{6}{*}{1} & 0.05 & -0.3258 & -0.3258 & -0.3259 & -0.3260 & -0.3262 & -0.3266 & -0.3277 & -0.3307 & -0.3383 & -0.3465 & -0.3627 \\
\hline & 0.1 & & -0.3258 & -0.3259 & -0.3259 & -0.3261 & -0.3266 & -0.3278 & -0.3307 & -0.3382 & -0.3465 & -0.3628 \\
\hline & 0.2 & & & -0.3259 & -0.3259 & -0.3261 & -0.3266 & -0.3276 & -0.3308 & -0.3382 & -0.3466 & -0.3631 \\
\hline & 0.3 & & & & -0.3259 & -0.3261 & -0.3266 & -0.3278 & -0.3304 & -0.3383 & -0.3466 & -0.3633 \\
\hline & 0.4 & & & & & -0.3262 & -0.3267 & -0.3282 & -0.3305 & -0.3385 & -0.3468 & -0.3636 \\
\hline & 0.5 & & & & & & -0.3267 & -0.3281 & -0.3310 & -0.3388 & -0.3473 & -0.3640 \\
\hline \multirow{6}{*}{0.5} & 0.05 & -0.3565 & -0.3565 & -0.3565 & -0.3568 & -0.3574 & -0.3581 & -0.3607 & -0.3662 & -0.3794 & -0.3925 & -0.4176 \\
\hline & 0.1 & & -0.3566 & -0.3566 & -0.3568 & -0.3571 & -0.3582 & -0.3608 & -0.3659 & -0.3791 & -0.3926 & -0.4177 \\
\hline & 0.2 & & & -0.3566 & -0.3568 & -0.3572 & -0.3583 & -0.3606 & -0.3665 & -0.3792 & -0.3927 & -0.4187 \\
\hline & 0.3 & & & & -0.3567 & -0.3574 & -0.3584 & -0.3609 & -0.3660 & -0.3795 & -0.3929 & -0.4190 \\
\hline & 0.4 & & & & & -0.3572 & -0.3585 & -0.3614 & -0.3661 & -0.3799 & -0.3935 & -0.4199 \\
\hline & 0.5 & & & & & & -0.3584 & -0.3615 & -0.3672 & -0.3808 & -0.3946 & -0.4212 \\
\hline \multirow{6}{*}{0.25} & 0.05 & -0.3686 & -0.3686 & -0.3686 & -0.3689 & -0.3697 & -0.3713 & -0.3750 & -0.3827 & -0.3998 & -0.4171 & -0.4485 \\
\hline & 0.1 & & -0.3687 & -0.3688 & -0.3691 & -0.3698 & -0.3715 & -0.3752 & -0.3823 & -0.3994 & -0.4172 & -0.4485 \\
\hline & 0.2 & & & -0.3688 & -0.3691 & $\begin{array}{l}-0.3699 \\
\end{array}$ & -0.3716 & -0.3750 & -0.3831 & -0.3997 & -0.4174 & -0.4497 \\
\hline & 0.3 & & & & -0.3690 & -0.3699 & -0.3718 & -0.3755 & -0.3828 & -0.4003 & -0.4181 & -0.4506 \\
\hline & 0.4 & & & & & -0.3698 & -0.3720 & -0.3764 & -0.3833 & -0.4015 & -0.4195 & -0.4506 \\
\hline & 0.5 & & & & & & -0.3718 & -0.3766 & -0.3850 & -0.4031 & -0.4218 & -0.4556 \\
\hline \multirow{6}{*}{0.125} & 0.05 & -0.3726 & -0.3726 & -0.3728 & -0.3732 & -0.3742 & -0.3762 & -0.3804 & -0.3892 & -0.4086 & -0.4281 & -0.4616 \\
\hline & 0.1 & & -0.3726 & -0.3727 & -0.3731 & -0.3740 & -0.3762 & -0.3808 & -0.3888 & -0.4083 & -0.4283 & -0.4638 \\
\hline & 0.2 & & & -0.3727 & -0.3732 & -0.3742 & -0.3764 & -0.3806 & -0.3899 & -0.4089 & -0.4290 & -0.4655 \\
\hline & 0.3 & & & & -0.3731 & -0.3743 & -0.3768 & -0.3813 & -0.3897 & -0.4103 & -0.4305 & -0.4676 \\
\hline & 0.4 & & & & & -0.3741 & -0.3772 & -0.3827 & -0.3909 & -0.4125 & -0.4334 & -0.4717 \\
\hline & 0.5 & & & & & & -0.3769 & -0.3834 & -0.3936 & -0.4155 & -0.4374 & -0.4772 \\
\hline \multirow{6}{*}{0} & 0.05 & -0.3747 & -0.3747 & -0.3749 & -0.3753 & -0.3765 & -0.3788 & -0.3836 & -0.3933 & -0.4148 & -0.4354 & -0.4729 \\
\hline & 0.1 & & -0.3747 & -0.3749 & -0.3754 & -0.3766 & -0.3790 & -0.3839 & -0.3937 & -0.4149 & -0.4363 & -0.4742 \\
\hline & 0.2 & & & -0.3749 & -0.3758 & -0.3772 & -0.3799 & -0.3851 & -0.3953 & -0.4176 & -0.4399 & -0.4803 \\
\hline & 0.3 & & & & -0.3755 & -0.3779 & -0.3813 & -0.3872 & -0.3984 & -0.4225 & -0.4460 & -0.4894 \\
\hline & 0.4 & & & & & -0.3775 & -0.3824 & -0.3897 & -0.4025 & -0.4292 & -0.4552 & -0.5031 \\
\hline & 0.5 & & & & & & -0.3820 & -0.3917 & -0.4068 & -0.4370 & -0.4661 & -0.5191 \\
\hline
\end{tabular}


TABLE 11 - Values of the influence coefficient $i_{3}$ at point $B$.

\begin{tabular}{|c|c|c|c|c|c|c|c|c|c|c|c|c|}
\hline & & & & & & & $\mathbf{a} / \mathbf{d}$ & & & & & \\
\hline $\mathrm{a} / \mathrm{c}$ & $2 \mathrm{a} / \mathrm{t}$ & 0.05 & 0.1 & 0.2 & 0.3 & 0.4 & 0.5 & 0.6 & 0.7 & 0.8 & 0.85 & 0.9 \\
\hline \multirow{6}{*}{1} & 0.05 & 0.3258 & 0.3258 & 0.3259 & 0.3258 & 0.3258 & 0.3258 & 0.3257 & 0.3256 & 0.3254 & 0.3253 & 0.3252 \\
\hline & 0.1 & & 0.3258 & 0.3258 & 0.3259 & 0.3258 & 0.3258 & 0.3257 & 0.3256 & 0.3254 & 0.3253 & 0.3252 \\
\hline & 0.2 & & & 0.3259 & 0.3259 & 0.3258 & 0.3258 & 0.3257 & 0.3255 & 0.3254 & 0.3253 & 0.3252 \\
\hline & 0.3 & & & & 0.3259 & 0.3259 & 0.3258 & 0.3257 & 0.3255 & 0.3254 & 0.3253 & 0.3252 \\
\hline & 0.4 & & & & & 0.3262 & 0.3259 & 0.3258 & 0.3256 & 0.3254 & 0.3253 & 0.3252 \\
\hline & 0.5 & & & & & & 0.3267 & 0.3262 & 0.3258 & 0.3256 & 0.3254 & 0.3254 \\
\hline \multirow{6}{*}{0.5} & 0.05 & 0.3565 & 0.3564 & 0.3565 & 0.3564 & 0.3563 & 0.3562 & 0.3560 & 0.3554 & 0.3550 & 0.3546 & 0.3542 \\
\hline & 0.1 & & 0.3565 & 0.3565 & 0.3565 & 0.3564 & 0.3563 & 0.3559 & 0.3554 & 0.3549 & 0.3546 & 0.3542 \\
\hline & 0.2 & & & 0.3566 & 0.3566 & 0.3565 & 0.3563 & 0.3560 & 0.3555 & 0.3549 & 0.3545 & 0.3542 \\
\hline & 0.3 & & & & 0.3568 & 0.3565 & 0.3562 & 0.3559 & 0.3554 & 0.3548 & 0.3544 & 0.3540 \\
\hline & 0.4 & & & & & 0.3572 & 0.3565 & 0.3560 & 0.3555 & 0.3548 & 0.3543 & 0.3539 \\
\hline & 0.5 & & & & & & 0.3584 & 0.3570 & 0.3560 & 0.3552 & 0.3546 & 0.3540 \\
\hline \multirow{6}{*}{0.25} & 0.05 & 0.3685 & 0.3686 & 0.3686 & 0.3684 & 0.3682 & 0.3679 & 0.3676 & 0.3668 & 0.3655 & 0.3652 & 0.3648 \\
\hline & 0.1 & & 0.3687 & 0.3687 & 0.3686 & 0.3684 & 0.3681 & 0.3675 & 0.3666 & 0.3655 & 0.3652 & 0.3648 \\
\hline & 0.2 & & & 0.3688 & 0.3686 & 0.3684 & 0.3680 & 0.3675 & 0.3666 & 0.3653 & 0.3649 & 0.3644 \\
\hline & 0.3 & & & & 0.3690 & 0.3685 & 0.3680 & 0.3674 & 0.3664 & 0.3650 & 0.3646 & 0.3641 \\
\hline & 0.4 & & & & & 0.3698 & 0.3686 & 0.3676 & 0.3665 & 0.3648 & 0.3642 & 0.3636 \\
\hline & 0.5 & & & & & & 0.3718 & 0.3692 & 0.3674 & 0.3653 & 0.3645 & 0.3637 \\
\hline \multirow{6}{*}{0.125} & 0.05 & 0.3726 & 0.3726 & 0.3726 & 0.3725 & 0.3722 & 0.3717 & 0.3710 & 0.3699 & 0.3681 & 0.3673 & 0.3665 \\
\hline & 0.1 & & 0.3726 & 0.3725 & 0.3724 & 0.3721 & 0.3717 & 0.3709 & 0.3697 & 0.3681 & 0.3672 & 0.3664 \\
\hline & 0.2 & & & 0.3727 & 0.3724 & 0.3721 & 0.3716 & 0.3708 & 0.3696 & 0.3677 & 0.3667 & 0.3655 \\
\hline & 0.3 & & & & 0.3730 & 0.3723 & 0.3715 & 0.3706 & 0.3693 & 0.3671 & 0.3660 & 0.3645 \\
\hline & 0.4 & & & & & 0.3741 & 0.3723 & 0.3709 & 0.3691 & 0.3666 & 0.3653 & 0.3635 \\
\hline & 0.5 & & & & & & 0.3769 & 0.3732 & 0.3705 & 0.3672 & 0.3655 & 0.3633 \\
\hline \multirow{6}{*}{0} & 0.05 & 0.3747 & 0.3747 & 0.3746 & 0.3743 & 0.3739 & 0.3733 & 0.3724 & 0.3707 & 0.3681 & 0.3661 & 0.3631 \\
\hline & 0.1 & & 0.3747 & 0.3745 & 0.3743 & 0.3738 & 0.3732 & 0.3722 & 0.3706 & 0.3679 & 0.3656 & 0.3624 \\
\hline & 0.2 & & & 0.3749 & 0.3742 & 0.3735 & 0.3726 & 0.3714 & 0.3695 & 0.3663 & 0.3638 & 0.3598 \\
\hline & 0.3 & & & & 0.3756 & 0.3738 & 0.3723 & 0.3706 & 0.3681 & 0.3641 & 0.3608 & 0.3556 \\
\hline & 0.4 & & & & & 0.3775 & 0.3740 & 0.3711 & 0.3676 & 0.3624 & 0.3582 & 0.3518 \\
\hline & 0.5 & & & & & & 0.3820 & 0.3756 & 0.3701 & 0.3631 & 0.3580 & 0.3500 \\
\hline
\end{tabular}

TABLE 12 - Values of the influence coefficient $i_{3}$ at point $C$.

\begin{tabular}{|c|c|c|c|c|c|c|c|c|c|c|c|c|}
\hline & & & & & & & $\mathrm{a} / \mathrm{d}$ & & & & & \\
\hline$a / c$ & $2 \mathrm{a} / \mathrm{t}$ & 0.05 & 0.1 & 0.2 & 0.3 & 0.4 & 0.5 & 0.6 & 0.7 & 0.8 & 0.85 & 0.9 \\
\hline \multirow{6}{*}{1} & 0.05 & 0.0000 & 0.0000 & 0.0000 & 0.0000 & -0.0001 & -0.0002 & -0.0004 & -0.0008 & -0.0013 & -0.0016 & -0.0021 \\
\hline & 0.1 & & 0.0000 & 0.0000 & 0.0000 & -0.0001 & -0.0002 & -0.0004 & -0.0008 & -0.0013 & -0.0016 & -0.0021 \\
\hline & 0.2 & & & 0.0000 & 0.0000 & -0.0001 & -0.0002 & -0.0004 & -0.0008 & -0.0013 & -0.0017 & -0.0022 \\
\hline & 0.3 & & & & 0.0000 & -0.0001 & -0.0002 & -0.0005 & -0.0008 & -0.0014 & -0.0017 & -0.0023 \\
\hline & 0.4 & & & & & 0.0000 & -0.0002 & -0.0005 & -0.0009 & -0.0014 & -0.0018 & -0.0024 \\
\hline & 0.5 & & & & & & 0.0000 & -0.0004 & -0.0009 & -0.0015 & -0.0019 & -0.0026 \\
\hline \multirow{6}{*}{0.5} & 0.05 & 0.0000 & 0.0000 & 0.0000 & 0.0000 & -0.0001 & -0.0003 & -0.0005 & -0.0008 & -0.0012 & -0.0015 & -0.0020 \\
\hline & 0.1 & & 0.0000 & 0.0000 & 0.0000 & -0.0001 & -0.0003 & -0.0005 & -0.0008 & -0.0012 & -0.0016 & -0.0020 \\
\hline & 0.2 & & & 0.0000 & 0.0000 & -0.0001 & -0.0003 & -0.0005 & -0.0008 & -0.0013 & -0.0016 & -0.0021 \\
\hline & 0.3 & & & & 0.0000 & -0.0001 & -0.0003 & -0.0006 & -0.0009 & -0.0014 & -0.0017 & -0.0023 \\
\hline & 0.4 & & & & & 0.0000 & -0.0003 & -0.0005 & -0.0009 & -0.0016 & -0.0019 & -0.0025 \\
\hline & 0.5 & & & & & & 0.0000 & -0.0005 & -0.0010 & -0.0017 & -0.0022 & -0.0028 \\
\hline \multirow{6}{*}{0.25} & 0.05 & 0.0000 & 0.0000 & 0.0000 & 0.0000 & -0.0001 & -0.0001 & -0.0002 & -0.0003 & -0.0005 & -0.0006 & -0.0008 \\
\hline & 0.1 & & 0.0000 & 0.0000 & 0.0000 & -0.0001 & -0.0001 & -0.0002 & -0.0003 & -0.0005 & -0.0007 & -0.0009 \\
\hline & 0.2 & & & 0.0000 & 0.0000 & -0.0001 & -0.0002 & -0.0003 & -0.0004 & -0.0006 & -0.0008 & -0.0010 \\
\hline & 0.3 & & & & 0.0000 & -0.0001 & -0.0002 & -0.0004 & -0.0005 & -0.0008 & -0.0010 & -0.0012 \\
\hline & 0.4 & & & & & 0.0000 & -0.0002 & -0.0004 & -0.0007 & -0.0010 & -0.0012 & -0.0016 \\
\hline & 0.5 & & & & & & 0.0000 & -0.0004 & -0.0007 & -0.0012 & -0.0015 & -0.0019 \\
\hline \multirow{6}{*}{0.125} & 0.05 & 0.0000 & 0.0000 & 0.0000 & 0.0000 & 0.0000 & 0.0000 & -0.0001 & -0.0001 & -0.0001 & -0.0002 & -0.0002 \\
\hline & 0.1 & & 0.0000 & 0.0000 & 0.0000 & 0.0000 & 0.0000 & -0.0001 & -0.0001 & -0.0002 & -0.0002 & -0.0002 \\
\hline & 0.2 & & & 0.0000 & 0.0000 & -0.0001 & -0.0001 & -0.0001 & -0.0002 & -0.0003 & -0.0003 & -0.0004 \\
\hline & 0.3 & & & & 0.0000 & -0.0001 & -0.0001 & -0.0002 & -0.0003 & -0.0004 & -0.0005 & -0.0006 \\
\hline & 0.4 & & & & & 0.0000 & -0.0001 & -0.0003 & -0.0004 & -0.0006 & -0.0007 & -0.0009 \\
\hline & 0.5 & & & & & & 0.0000 & -0.0002 & -0.0004 & -0.0007 & -0.0009 & -0.0011 \\
\hline
\end{tabular}

